\title{
ASSOCIAÇÃO DA COGNIÇÃO COM FATORES SOCIOEDEMOGRÁFICOS E QUALIDADE DE VIDA EM CRIANÇAS PORTADORAS DE FISSURA OROFACIAL NÃO SINDRÔMICA
}

\author{
ASSOCIATION OF COGNITION WITH SOCIODEMOGRAPHIC FACTORS AND \\ QUALITY OF LIFE IN CHILDREN WITH NON-SYNDROMIC OROFACIAL \\ FISSURE
}

Ergellis Victor Cavalcanti de Lima, Ândrea Gomes Salles, Dayse da Silva Chaves, Fernanda Baia da
Costa, Aloiso Sampaio Souza, Rossana Vanessa Dantas de Almeida Marques Universidade Federal do Maranhão - UFMA

\begin{abstract}
Thisis a quantitative study with 67 children, between 05 and 11 yearsold, grouped as follows: A1 (patients with cleftlipandpalate; $n=21$ ), A2 (normorreative childrenlinkedto a privateschool; $n=23$ ) and $A 3$ ( normorreative children linked to the public school; $n=$ 23), A1 beingthe case groupand $A 2$ and $A 3$ thecontrolgroups. Anamnesis, cognitive evaluation, medical records verification, quality of life analysis and oral health quality assessment were made with the need toob tain correlations. The sample distribution was similar too ther studies. In A1, a fissure transforms the numerical relation ship with intelligence belo wtheaverage $(n=5)$. The Group A2 showed higher socioeconomic scores in contrastto $A 1$ and $A 3$, un related to cognition. In $A 2$, the social disability do mainisaffected with below-averageintelligence $(p=$ 0.005), which does notoccur in theother do mainsof oral health quality in thesa megroupand $A 1$ and $A 2$. In the quality of life $A 1$, there achdomain shows number slessthan $A 2$ and $A 3$, with no correlation with the type sofintelligence; with other domains similar too ther studies. There fore, thecognition of children with nonsyndromic orofacial cleft does not show a significant relations hipwith quality of life, qualityof oral heal thand socioeconomic profile. There fore, theim portance of multiprofession altreatmentand its impacton the normalization of the life of a fissured child is evident.
\end{abstract}

Key words: CleftLip; Qualityoflife; Cognition; Kid.

\section{Resumo}

Trata-se de um estudo quantitativo com 67 crianças, entre 05 e 11 anos, agrupadas da seguinte forma: A1 (portadores de fissura labiopalatina; $n=21$ ), $A 2$ (crianças normorreativas vinculadas a escola privada; $n=23$ ) e A3 (crianças normorreativas vinculadas a escola pública; $n=23$ ), sendo A1 o grupo caso e A2 e A3 os grupos controles. Realizaram-se anamnese, avaliação cognitiva, verificação de prontuários, análise da qualidade de vida e avaliação da qualidade de saúde oral; com a finalidade de obter correlações. A distribuição da amostra foi semelhante a outros estudos. Em A1, a fissura transforame obteve relação numérica com a inteligência abaixo da média $(n=5)$. O grupo A2 apresentou maiores pontuações socioeconômicas em contraponto a A1 e A3, sem relação com a cognição. No A2 o domínio da incapacidade social obteve relevância com a inteligência abaixo da média( $(p=0,005)$, o que não se observou nos demais domínios de qualidade de saúde bucal do mesmo grupo e do A1 e A2. Na qualidade de vida do $A 1$, o domínio da autonomia apresentou números menores que o A2 e A3, sem correlação com os tipos de inteligência; com os demais domínios semelhantes a outros estudos. Logo, a cognição das crianças portadoras de fissura orofacial não sindrômica não apresentou relação significativa com qualidade de vida, qualidade de saúde oral e perfil socioeconômico. Evidencia-se, portanto, a importância do tratamento multiprofissional e o seu impacto na normatização da vida de uma criança fissurada.

Palavras chave: Fenda Labial; Qualidade de vida; Cognição; Criança. 


\section{Introdução}

A priori, a fissura orofacial adentra como a malformação craniofacial mais comum a âmbito mundial', resultando de anomalias entre a 4으 e 12 o semana de gestação ${ }^{2}$. Para tal, possui como etiologia o seu caráter multifatorial, tendo em vista a variabilidade genética e ambiental envolvidas, englobando entidades como infecções perinatais, deficiência nutricional, exposição teratogênica, tabagismo ${ }^{3}$, etilismo, idade materna avançada, bem como a expressão gênica diversa ${ }^{4}$. Dessa forma, afetando um por mil nascidos vivos, refere-se ao acometimento do lábio, do palato ou fenda labial e palatina (FLP), associado ou não a uma síndrome concomitante ${ }^{5}$, o qual pode variar de acordo com a raça, a população e a etnia ${ }^{6,7}$.

$\mathrm{Na}$ esteira desse raciocínio, o tratamento efetivo, somado ao correto diagnóstico são primordiais, já que esses pacientes são acometidos por problemas na função mastigatória, má oclusão, fonética, infecções recorrentes das vias aéreas, aspectos faciais estéticos e progressão psicossocial. Somado a isso, na infância, esses indivíduos estão submetidos a adversidades comportamentais, cognitivas e dinâmicas ${ }^{8}$, que são modificadas mediante as demandas e esforços do contexto em que está inserido. Assim, averiguar a cognição, isto é, a capacidade de mutualismo entre o corpo e o cérebro na construção do ambiente que o rodeia e a sua imagem é essencial, especialmente ao se comparar as crianças normorreativas e as divergentes variáveis socioeconômicas.

Destarte, torna-se impreterível apontar a importância da qualidade de vida envolvida, pois amplia conceitos individuais, não raro esquecidos, direcionando para as limitações existentes. Desse modo, realiza uma avaliação do bem-estar social inserida em uma percepção cultural ${ }^{9}$. Agregado a isso, a qualidade de vida, no que tange a saúde oral direciona as melhores condutas em centros especializados, já que é capaz de fornecer informações acerca dos impactos que essas alterações faciais alcançam ${ }^{10}$.

Embora a fissura orofacial represente uma alta incidência estatística, nas produções literárias são reduzidos os estudos acerca da análise cognitiva, da qualidade de vida, bem como da relação entre essas variáveis em contextos sociais diferentes, especialmente no público infantil. Por conseguinte, a elaboração de pesquisas com essa temática direciona uma contribuição expressiva nos programas de reabilitação e prevenção, oportunizando uma incorporação educacional, a fim de reduzir os impactos da malformação em todas as suas dimensões.

Logo, se alvidrou analisar a associação da condição cognitiva com os fatores sociodemográficos e qualidade de vida em crianças portadoras de fissura orofacial não sindrômicas, comparando, em um estudo caso controle, com crianças normorreativas que não a possuem.

\section{Metodologia}

De acordo com as recomendações do Conselho Nacional de Saúde (CNS) e a resolução $466 / 201211$, os aspectos éticos fundamentais, como a equidade, a justiça, a não maleficência, a beneficência e a autonomia foram contemplados rigorosamente desde a idealização e planejamento a execução das atividades.

Para tal, institui-se um estudo de caráter observacional, transversal e quantitativo, mediante os procedimentos éticos da cientificidade com a aprovação do comitê de Ética em pesquisa com seres humanos da Universidade Federal do Maranhão (CAAE 96461418100005087).

Concomitantemente, obteve-se a formalização e concordância dos pais responsáveis pelas crianças, utilizando-se o Termo de Assentimento Livre e Esclarecido e o Termo de Consentimento Livre Esclarecido (TCLE).

Foram contemplados na amostra 67 crianças, de ambos os sexos, compreendidas na faixa etária entre 05 e 11 anos. De tal forma, agrupou-se os seguintes grupos: A1 (portadores de fissura labiopalatina; $\mathrm{n}=21$ ); $A 2$ (crianças normorreativas vinculadas a escola privada; $n=$ 23) e A3 (crianças normorreativas vinculadas a escola pública; $n=23$ ), constituindo $A 1$ o grupo caso e grupos controle A2 e A3.

As crianças acometidas por fissura eram assistidas pelo Centrinho Imperatriz (Maranhão, Brasil) instituição que realiza assistência integral sem fins lucrativos, com abrangência geográfica para as regiões do sul do Maranhão, norte do Tocantins e sul do Pará. 
Agendadas para as diferentes especialidades relacionadas ao tratamento sequencial, as crianças assistidas pelo Centrinho Imperatriz (A1) foram selecionadas por conveniência, independente da fase cirúrgica reparadora. Paracompor A2 e A3 a seleção da amostra também ocorreu por conveniência, conforme o estabelecimento de ensino de vínculo da criança, com pareamento para faixa etária e sexo. Como critérios de exclusão, crianças com diagnóstico de alterações sensoriais, psiquiátricas, sindrômica ou neurológica não foram incluídas na amostra.

Tendo em vista a condução adequada da execução da pesquisa, realizaram-se os seguintes procedimentos: análise de prontuários, anamnese, análise socioeconômica, análise cognitiva, avaliação da qualidade de vida e avaliação da qualidade de saúde oral.

A anamnese forneceu uma relação pesquisador-criança satisfatória, permitindo a aplicação de um questionário estruturado contendo informações como a escolaridade, a idade, a etnia, além de adentrar na aplicação dos demais questionários validados. Somado a isso, a análise de prontuários no Centrinho Imperatriz integralizou corretamente as informações relativas ao tipo de fissura labiopalatina, classificadas de acordo com Spina, bem como forneceu o histórico de realizações de cirurgias reparadoras nos portadores de fissura labiopalatina.

Originado da Associação Brasileira de empresas de pesquisa (ABEP), empregou-se o critério de classificação econômico Brasil para a avaliação socioeconômica da amostra, questionando acerca no nível de instrução escolar dos responsáveis, da presença de serviços públicos na comunidade, bem como da presença de bens materiais no domicílio. Tais dados, possibilitou as seguintes denominações e pontuações: classe A (45 a 100 pontos); classe B1 (38 a 44); classe B2 (29 e 37); classe C1 (23 a 28); classe C2 (17 a 22) e, por último, classe D-E (16 a $0)$.

Para a quantificação da avaliação e desenvolvimento cognitivo, foram utilizados os testes padronizados da coleção R-2, o qual comporta a validação restrita a faixa etária de 05 a 11 anos, constituído por 30 questões, definindo para o presente estudo três categorias: Acima da média, média e abaixo da média. Para tal, ausentes de interferências externas, foram feitas em ambientes contemplativos.

O questionário validado denominado AutoquestionnaireQualité de Vie Enfant Imagé(AUQEI) foi utilizado para a análise da qualidade de vida da amostra, o qual permite a faixa etária de 04 a 12 anos, e atribui 4 níveis de sentimentos por parte das crianças diante dos 26 questionamentos propostos: muito infeliz, infeliz, feliz e muito feliz. Por conseguinte, tendo em consideração os domínios da autonomia, lazer, funções e família, os resultados foram obtidos por meio da pontuação das respostas nas variações de 0 a 3.

O teste Oral Health Impact Profile (OHIP 14) foi operado com a finalidade de dimensionar a qualidade de vida relacionada a saúde oral. Tal escolha fora realizada tendo em vista o seu reconhecimento e magnitude no âmbito internacional para a análise das condições bucais, mediante seus efeitos adversos no bem-estar dos indivíduos.

Nesse sentido, atribui as suas 14 questões o papel de indagar, nos últimos 12 meses, a presença de algum incidente social devido a sua condição oral. Dessa forma, apresenta para cada dois itens do seu questionário os presentes domínios: dor física; limitação funcional; incapacidade física; desconforto psicológico; incapacidade psicológica; incapacidade social e desvantagem social.

Por fim, os dados foram analisados no programa StatisticalPackage For Social Sciences (SPSS 19.0 for Windows), mediante armazenamento em planilhas do Microsoft Excel, com nível de confiança de $95 \%$. Utilizaram-se detalhamento de percentuais e frequências numéricas, desvio padrão, médias e em seguimento, foram empregados estatística inferencial por meio do teste Qui-quadrado e do teste não paramétricoKruskal-Wallis, comparando as variáveis independentes, seguido de teste post-hoc de Dunn quando permitido.

\section{Resultados}

O presente estudo investigou uma amostra composta por 67 crianças, seccionando em 21 crianças portadoras de fissura orofacial não sindrômica (A1, $n=21 ; 31.3 \%)$, crianças vinculadas a escola privada ( $A 2, n=23 ; 34.3 \%)$ e crianças vinculadas a escola pública $(A 3 ; n=23$; 
$34.3 \%)$. A idade média alcançada dos grupos foi de $7,9( \pm 2.33)$ para $A 1 ; 7,47( \pm 1.99)$ para $A 2$ e 8,6 ( \pm 2.56$)$ para A3.

Das crianças portadoras de fissuras orofaciais, que compõem o $A 1,19.05 \%(n=4)$ são provenientes de escola privada e $80.95 \%$ (n =17) são provenientes de escola pública. Os procedimentos cirúrgicos cujas crianças já foram expostas foram palatoplastia (80.95\%), queiloplastia (61.90\%) e enxertia óssea (19.04\%). Além disso, tem-se como etnia predominante a parda $(n=14,66.7 \%)$, seguida da etnia branca $(n=$ $6,28.6 \%)$ e $4.8 \%$ indígena.

A categorização do nível de cognição contou com o predomínio da inteligência média em todos os grupos. Entretanto, ao se comparar, o grupo A1 obteve $47.6 \% \quad(n=10)$, o grupo A2 $56.5 \%(n=13)$ e o grupo $A 343.4 \%(n=10)$.

A seguir, a tabela 1 descreve a associação entre a cognição e as demais variáveis no grupo A1. Tratando-se do gênero, compreende-se o predomínio do sexo masculino (57.1\%), englobados principalmente na inteligência média. De forma análoga, dos $42,9 \%$ pacientes do sexo feminino $(n=9)$, a inteligência média prevaleceu $(n=5)$, sem no entanto, haver um valor estatístico significante $(P=0.269)$.

No que diz respeito aos tipos de fissuras avaliadas, a transforame $(n=10)$ sobressaiu, categorizada em maior número na inteligência abaixo da média. Em sequência, a fissura pósforame $(n=7)$ obteve destaque na inteligência média (57.1\%), seguida pela fissura pré-forame $(n=4)$ a qual obteve valor majoritário também na inteligência média.

No que tange a classificação socioeconômica do grupo A1, nota-se o predomínio da classe C-2 $(n=9)$. Para tal, as classes ditas B1, C1 e C2 estiveram presentes notavelmente na inteligência média, em contraposição a inteligência abaixo da média que sobressaiu na classe A e classe $D-E$, com $p=0.441$.

Acerca da classificação da qualidade de vida do grupo de fissurados, tem-se um predomínio da boa pontuação $(n=15)$, dos quais $46.7 \%$ são provenientes de uma análise cognitiva classificada como inteligência média. Em continuação de tal quesito, as médias que pontuaram os seus domínios demonstraram que a função e o lazer adentraram especialmente na correlação com a inteligência acima da média, enquanto que a família e autonomia na inteligência média.

Por sua vez, os domínios da qualidade de saúde oral sinalizaram maiores médias para a inteligência abaixo da média nos seguintes quesitos: dor física, desconforto psicológico, incapacidade física, incapacidade psicológica e desvantagem social. A cognição acima da média, por seu valor, contou com a presença prevalente do domínio da limitação funcional, em contraponto ao domínio da incapacidade social, o qual compartilhou valores iguais para a inteligência abaixo da média e inteligência média.

Tabela 1. Correlação entre o nível cognitivo com as variáveis sociodemográficas, qualidade de vida e qualidade de saúde oral nas crianças portadoras de fissura orofacial (A1).

\begin{tabular}{|c|c|c|c|c|c|c|c|}
\hline \multirow{2}{*}{ VARIÁVEIS } & \multicolumn{2}{|c|}{ Acima da média } & \multicolumn{2}{|r|}{ Média } & \multicolumn{2}{|c|}{ Abaixo da média } & \multirow[t]{2}{*}{ P-valor* } \\
\hline & $\mathbf{n}$ & $\%$ & $\mathbf{n}$ & $\%$ & $\mathbf{n}$ & $\%$ & \\
\hline \multicolumn{8}{|l|}{ Sexo } \\
\hline Masculino & 3 & $25 \%$ & 5 & $41.7 \%$ & 4 & $33.3 \%$ & $0,269^{a}$ \\
\hline Feminino & 0 & $0 \%$ & 5 & $55.6 \%$ & 4 & $44.4 \%$ & \\
\hline \multicolumn{8}{|c|}{ Tipos de Fissuras } \\
\hline Pré-forame & 1 & $25 \%$ & 2 & $50 \%$ & 1 & $25 \%$ & $0,839^{a}$ \\
\hline Pós-forame & 1 & $14.3 \%$ & 4 & $57.1 \%$ & 2 & $28.6 \%$ & \\
\hline Transforame & 1 & $10 \%$ & 4 & $40 \%$ & 5 & $50 \%$ & \\
\hline \multicolumn{8}{|c|}{$\begin{array}{c}\text { Classificação socioeconômica } \\
\text { (ABEP) }\end{array}$} \\
\hline Classe A & 0 & $0 \%$ & 0 & $0 \%$ & 1 & $100 \%$ & $0,441^{\mathrm{a}}$ \\
\hline Classe B1 & 0 & $0 \%$ & 1 & $100 \%$ & 0 & $0 \%$ & \\
\hline Classe C1 & 0 & $0 \%$ & 3 & $75 \%$ & 1 & $25 \%$ & \\
\hline
\end{tabular}


...continuação

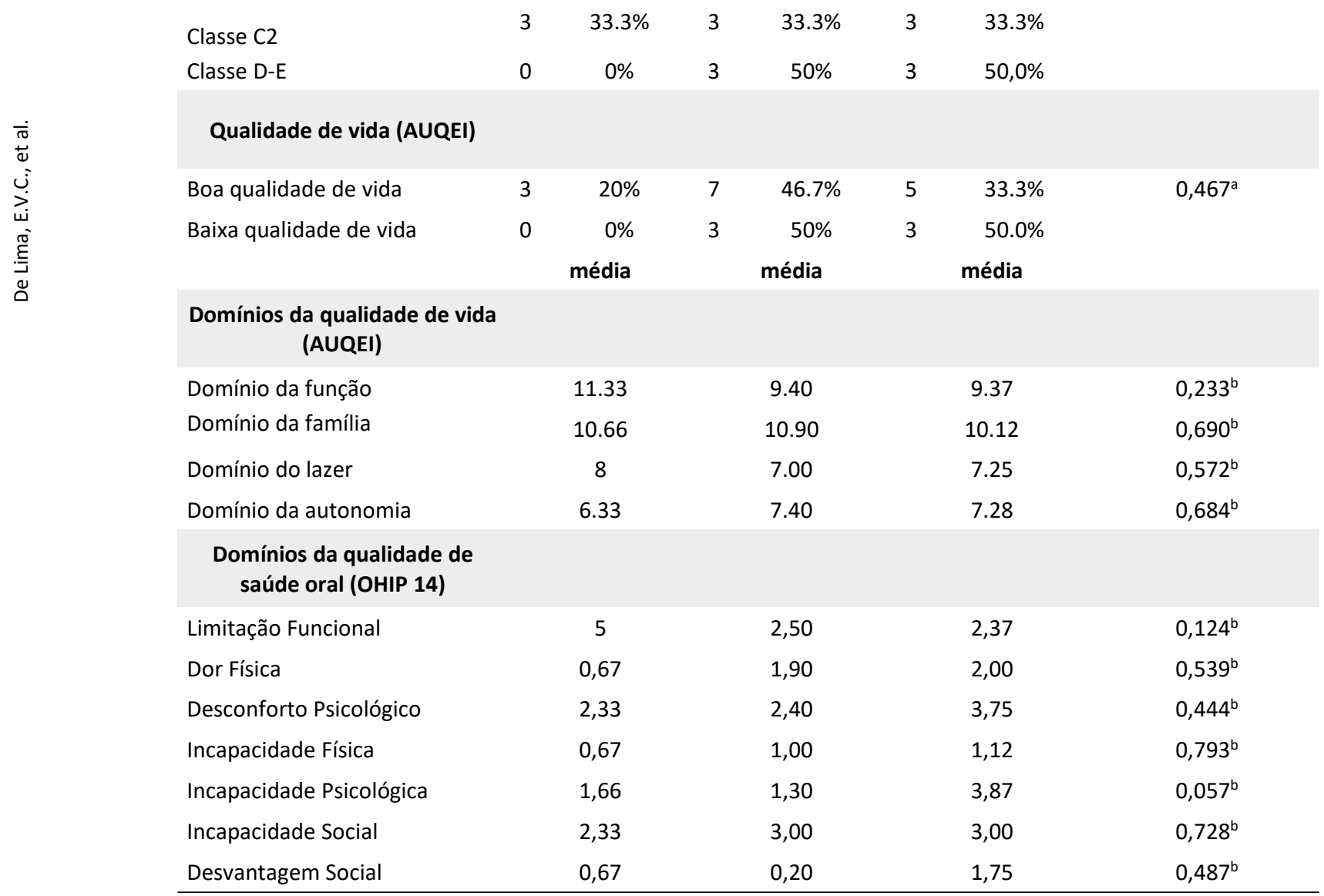

${ }^{\mathrm{a}}$ Teste Qui-quadrado de homogeneidade.

${ }^{\mathrm{b}}$ Teste Kruskal-Wallis

Das crianças provenientes da instituição privada (A3), houve predomínio da etnia branca com 56.5\% ( $n=13)$. Para tal, se compôs, em sua maioria, do sexo masculino, sobrelevando na inteligência média (tabela 2). Além disso, atentando-se a classificação socioeconômica, a ausência das classes C2 e D-E, bem como a prevalência da classe B2, especialmente associada a inteligência média é observada.

No que concerne a pontuação da qualidade de vida do grupo A2, a contagem tida como boa é predominante, particularmente correlacionado a inteligência média (47.4\%). Complementando essa categoria, a média do domínio da função
(10.69) obteve maior valor na inteligência média. Os domínios da família e lazer, por sua vez, estiveram maiores na inteligência abaixo da média e o domínio da autonomia na inteligência acima da média (10.25), sem no entanto obtiver significância estatística.

A qualidade de saúde oral, ainda no grupo $A 2$, atribui ausência de pontuação no domínio da desvantagem social, incapacidade física e limitação funcional. Ademais, há p-valor(0.005) significativo, na relação entre o domínio da incapacidade social e a inteligência abaixo da média. 
Tabela 2. Correlação entre o nível cógnito com as variáveis sociodemográficas, qualidade de vida e qualidade de saúde oral em crianças normorreativas vinculadas a escola privada (A2).

\begin{tabular}{|c|c|c|c|c|c|c|c|}
\hline \multirow{2}{*}{ VARIÁVEIS } & \multicolumn{2}{|c|}{ Acima da média } & \multicolumn{2}{|r|}{ Média } & \multicolumn{2}{|c|}{ Abaixo da média } & \multirow[t]{2}{*}{ P-valor* } \\
\hline & n & $\%$ & $\mathbf{n}$ & $\%$ & $\mathbf{n}$ & $\%$ & \\
\hline \multicolumn{8}{|l|}{ Sexo } \\
\hline Masculino & 4 & $33,3 \%$ & 6 & $50 \%$ & 2 & $16,7 \%$ & $0,361^{a}$ \\
\hline Feminino & 4 & $36,4 \%$ & 7 & $63,6 \%$ & 0 & $0 \%$ & \\
\hline \multicolumn{8}{|c|}{$\begin{array}{c}\text { Classificação socioeconômica } \\
\text { (ABEP) }\end{array}$} \\
\hline Classe A & 1 & $100 \%$ & 0 & $0 \%$ & 0 & $0 \%$ & $0,217^{\mathrm{a}}$ \\
\hline Classe B1 & 2 & $25,0 \%$ & 6 & $75 \%$ & 0 & $0 \%$ & \\
\hline Classe B2 & 2 & $20 \%$ & 6 & $60 \%$ & 2 & $20 \%$ & \\
\hline Classe C1 & 3 & $75,0 \%$ & 1 & $25 \%$ & 0 & $0 \%$ & \\
\hline \multicolumn{8}{|c|}{ Qualidade de vida (AUQEI) } \\
\hline Boa qualidade de vida & 8 & $42,1 \%$ & 9 & $47,4 \%$ & 2 & $10,5 \%$ & $0,155^{\mathrm{a}}$ \\
\hline \multirow[t]{2}{*}{ Baixa qualidade de vida } & 0 & $0 \%$ & 4 & $100 \%$ & 0 & $0 \%$ & \\
\hline & & média & & Média & & média & \\
\hline \multicolumn{8}{|c|}{$\begin{array}{l}\text { Domínios da qualidade de vida } \\
\text { (AUQEI) }\end{array}$} \\
\hline Domínio da função & & 10,37 & & 10,69 & & 10,50 & $0,915^{b}$ \\
\hline Domínio da família & & 11,12 & & 10,31 & & 11,50 & $0,994^{b}$ \\
\hline Domínio do lazer & & 8,12 & & 7,92 & & 9,00 & $0,365^{b}$ \\
\hline Domínio da autonomia & & 10,25 & & 9,15 & & 7,50 & $0,125^{b}$ \\
\hline \multicolumn{8}{|c|}{$\begin{array}{l}\text { Domínios da qualidade de } \\
\text { saúde oral (OHIP 14) }\end{array}$} \\
\hline Limitação Funcional & & 0,00 & & 0,00 & & 0,00 & - \\
\hline Dor Física & & 0,50 & & 0,62 & & 3,00 & $0,063^{b}$ \\
\hline Desconforto Psicológico & & 0,13 & & 0,08 & & 0,50 & $0,273^{b}$ \\
\hline Incapacidade Física & & 0,00 & & 0,00 & & 0,00 & - \\
\hline Incapacidade Psicológica & & 0,00 & & 0,15 & & 0,00 & $0,681^{b}$ \\
\hline Incapacidade Social & & 0,00 & & 0,00 & & 1,00 & $0,005^{b}$ \\
\hline Desvantagem Social & & 0,00 & & 0,00 & & 0,00 & - \\
\hline
\end{tabular}

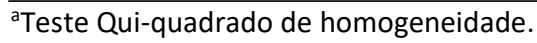

b Teste Kruskal-Wallis.

O grupo A3 é constituído em sua maioria por $47.8 \%$ de pardos $(n=11)$. A tabela 3 demonstra o vínculo entre a cognição da criança e as variáveis, tendo o sexo feminino em seu domínio, dos quais $50 \%$ estão correspondentes a inteligência média. Outrossim, a classe socioeconômica aponta para ausência da classe A e B1, com dominância da classe $\mathrm{C} 2$, sobretudo na inteligência média ( $p=0.536)$.

Em continuidade, a qualidade de vida do grupo A3 alcançou valores maiores na categorização da boa qualidade do AUQEI ( $N=19)$, 
interligado de forma numérica igual tanto na inteligência média quanto na inteligência abaixo da média. Os seus domínios, por seu lado, lograram médias maiores na cognição acima da média. Além disso, dentre os domínios da qualidade de saúde oral quantificados pelo OHIP 14 , tem-se como domínio com maior média a dor física, tendo correlação numérica evidente com a inteligência média, sem, no entanto, apresentar p-valor considerável.

Tabela 3. Correlação entre nível cognitivo com variáveis sociodemográficas, qualidade de vida e qualidade de saúde oral em crianças normorreativas vinculadas a escola pública (A3).

\begin{tabular}{|c|c|c|c|c|c|c|c|}
\hline \multirow{2}{*}{ VARIÁVEIS } & \multicolumn{2}{|c|}{ Acima da média } & \multicolumn{2}{|r|}{ Média } & \multicolumn{2}{|c|}{ Abaixo da média } & \multirow[t]{2}{*}{ p-valor* } \\
\hline & $\mathbf{n}$ & $\%$ & $\mathbf{n}$ & $\%$ & $\mathbf{n}$ & $\%$ & \\
\hline \multicolumn{8}{|l|}{ Sexo } \\
\hline Masculino & 4 & $36.4 \%$ & 4 & $36.4 \%$ & 3 & $27.3 \%$ & $0,264^{\mathrm{a}}$ \\
\hline Feminino & 1 & $8.3 \%$ & 6 & $50 \%$ & 5 & $41.7 \%$ & \\
\hline \multicolumn{8}{|c|}{$\begin{array}{c}\text { Classificação socioeconômica } \\
\text { (ABEP) }\end{array}$} \\
\hline Classe B2 & 1 & $33 \%$ & 1 & $33.3 \%$ & 1 & $33.3 \%$ & $0,536^{\mathrm{a}}$ \\
\hline Classe C1 & 1 & $20 \%$ & 3 & $60 \%$ & 1 & $20 \%$ & \\
\hline Classe C2 & 3 & $33.3 \%$ & 4 & $44.4 \%$ & 2 & $22.2 \%$ & \\
\hline Classe D-E & 0 & $0 \%$ & 2 & $33.3 \%$ & 4 & $66.7 \%$ & \\
\hline \multicolumn{8}{|c|}{ Qualidade de vida (AUQEI) } \\
\hline Boa qualidade de vida & 4 & $21.1 \%$ & 9 & $47.4 \%$ & 6 & $31.6 \%$ & $0,695^{\mathrm{a}}$ \\
\hline \multirow[t]{2}{*}{ Baixa qualidade de vida } & 1 & $25 \%$ & 1 & $25 \%$ & 2 & $50 \%$ & \\
\hline & & média & & Média & & média & \\
\hline \multicolumn{8}{|c|}{$\begin{array}{l}\text { Domínios da qualidade de vida } \\
\text { (AUQEI) }\end{array}$} \\
\hline Domínio da função & & 10.80 & & 10.50 & & 9.50 & $0.309^{b}$ \\
\hline Domínio da família & & 11.20 & & 10.80 & & 10.62 & $0.964^{b}$ \\
\hline Domínio do lazer & & 8.20 & & 7.70 & & 7.50 & $0.550^{\mathrm{b}}$ \\
\hline Domínio da autonomia & & 8.40 & & 7.80 & & 6.87 & $0.310^{\mathrm{b}}$ \\
\hline \multicolumn{8}{|c|}{$\begin{array}{l}\text { Domínios da qualidade de } \\
\text { saúde oral (OHIP 14) }\end{array}$} \\
\hline Limitação Funcional & & 0.40 & & 0.30 & & 0.25 & $0.803^{b}$ \\
\hline Dor Física & & 2.00 & & 1.00 & & 1.50 & $0.495^{b}$ \\
\hline Desconforto Psicológico & & 0.00 & & 0.20 & & 0.38 & $0.439^{b}$ \\
\hline Incapacidade Física & & 0.00 & & 0.20 & & 0.13 & $0.569^{b}$ \\
\hline Incapacidade Psicológica & & 0.40 & & 0.20 & & 0.00 & $0.412^{\mathrm{b}}$ \\
\hline Incapacidade Social & & 0.00 & & 0.00 & & 0.38 & $0.141^{b}$ \\
\hline Desvantagem Social & & 0.00 & & 0.20 & & 0.00 & $0.522^{\mathrm{b}}$ \\
\hline
\end{tabular}

Teste Qui-quadrado de homogeneidade.

b Teste Kruskal-Wallis. 


\section{Discussão}

No que se refere a distribuição por etnias das crianças portadoras de fissura orofacial, o presente estudo demonstrou, numericamente, consonância com diversas investigações epidemiológicas ${ }^{11,12}$. Logo, tal fato certifica e aponta para taxas menores de acometimento em indivíduos negros, em contraposição à etnia branca, razão ainda desconhecida.

Por sua vez, a distribuição da fissura transforame do A1 (47.63\%), bem como o predomínio do gênero masculino (57.1\%) são evidenciados por outros importantes estudos ${ }^{13,14}$, corroborando que a amostra representa o perfil típico da fissura orofacial não sindrômica. Levando em consideração a susceptibilidade do gênero masculino, tem-se como teoria principal o impacto do MSX1, um gene o qual está fixo no cromossomo $4^{15}$.

Dentre as diversas apresentações da fissura orofacial, entende-se que a fissura transforame é tida como a malformação mais grave, devido a sua maior extensão, o envolvimento de estruturas e, por conseguinte, com significantes comprometimentos estéticos e funcionais ${ }^{16}$. Embora, de forma numérica a correlação entre tal apresentação com a cognição tida como abaixo da média tenha prevalecido, compreendese que há ausência de inter-relação comparativamente as demais fissuras, já que o valor estatístico foi insignificante.

Em continuidade, abordagens literárias, as quais embora utilizaram outras ferramentas para a análise cognitiva, apontaram para as maiores pontuações em grupos da fissura pré-forame com ausência de significância estatística ${ }^{17}$. No presente estudo, percentualmente tal grupo, numericamente, apresentou melhores pontuações, já que $50 \%$ foram enquadrados em inteligência acima da média e $25 \%$ abaixo da média. No entanto, de forma semelhante também não apresentou relevância estatística.

Partindo do conceito de que cognição envolve um caráter multidimensional ${ }^{18}$, o tipo de escola adentra como um fator importante. Isso porque em estudos ${ }^{19,20}$, crianças provenientes de escolas particulares obtiveram melhores pontuações nas diversas habilidades. Comparativamente, nesse estudo se observa que as crianças provenientes de escola privada, apresentaram maior representatividade na inteligência dita acima da média( $\mathrm{N}=8)$, superando o grupo $A 1$ e o grupo $A 3$, mas sem significância. Essas informações demonstram o papel crucial envolvido nos contextos escolares e até que ponto a estimulação do meio influencia no andamento da construção do indivíduo ${ }^{21}$.

Em pesquisas a condição socioeconômica norteia os aspectos mais gerais da inteligência ${ }^{22}$. O impacto das variáveis socioeconômicas na inteligência se dá, possivelmente, pela estimulação cognitiva oferecida às crianças pelos seus cuidadores parentais, a qual pode apresentar diferenças de acordo com a renda da família. No estudo apresentado, as crianças oriundas do grupo A2 obtiveram maiores pontuações comparativamente ao grupo A1 e A3, porém não houve uma relação significativa a ponto de estabelecer uma relação casuística entre os diversos tipos de inteligência e a renda familiar.

Diversos são os estudos que apontam para uma qualidade de vida deficitária em crianças portadoras de fissura orofacial ${ }^{23,24,25}$. No entanto, é escasso a sua relação (qualidade de vida dita boa ou baixa) com a cognição. $\mathrm{Na}$ análise, observa-se que os três grupos não apresentaram significância estatísticas nesse quesito, apontando que a criança portadora de fissura labiopalatina é capaz de manter normatização, desde que bem assistidas.

Ao se comparar os domínios da qualidade de vida, nota-se que tanto no grupo $A 1, A 2$ e $A 3$ os domínios da família, função e lazer não apresentaram divergências importantes entre si, condizente com análises de outros autores ${ }^{26}$. 0 domínio da autonomia, embora continue sem dados estatísticos significantes ao ser relacionada com a inteligência acima da média, inteligência média e inteligência abaixo da média; possui no grupo $\mathrm{A} 1$ as menores médias. Logo, não se pode afirmar no presente estudo que a interferência da cognição na qualidade de vida é divergente de uma crianças portadora de fissura e de uma criança normorreativa, seja de escola privada ou pública.

Em pesquisa anterior ${ }^{27}$, sem grupos controle, destrinchou-se que alterações no domínio da dor física, desconforto psicológico, incapacidade psicológica e incapacidade social na qualidade de saúde oral foram observadas em crianças portadoras de fissura. Logo, contata-se que os domínios do desconforto psicológico, da 
incapacidade física, da incapacidade psicológica, da incapacidade social e da desvantagem social estão mais relacionadas a inteligência abaixo da média, demonstrando uma possível relação. Por outro lado, os domínios da limitação funcional e da dor física não seguiram esse padrão.

A análise da qualidade da cavidade oral se torna mais evidente no grupo $A 2$, que apresenta valor significativo ( $P$-valor 0,005$)$ no que tange a relação da incapacidade social e o tipo de inteligência. Ou seja, as crianças provenientes da instituição privada sofrem menos interferência nesses quesitos, ao se contrapor os grupos $\mathrm{A} 1 \mathrm{e}$ A3. Isso pode ser explicado pelas divergências socioeconômicas dos grupos, bem como das divergências anatomofuncionais.

\section{Conclusão}

As grandes dificuldades na investigação residiram na captação das crianças fissuradas devido à dificuldade de locomoção até o centro especializado para a realização das análises, somado a vulnerabilidade socioeconômica e o abandono ao tratamento por parte de alguns pacientes. Além disso, englobar testes cognitivos concomitantes que encaixem no perfil da faixa etária proposta foi uma dificuldade superada.

Avaliar a associação da cognição com fatores sociodemográficos, qualidade de vida e qualidade de saúde oral em crianças portadoras de fissura orofacial não sindrômica, possibilita a equipe multidisciplinar, atuante na reabilitação dos indivíduos com fissura labiopalatina, uma reflexão quanto ao direcionamento do tratamento, pois identificar as correlações permite rever as propostas de intervenção oferecidas, com a finalidade de contribuir para a melhoria da qualidade da assistência e, consequentemente, a promoção da saúde.

Conclui-se, portanto, que as crianças deste grupo não apresentaram evidencias estatísticas na correlação entre a inteligência média e as diversas variedades analisadas. Logo, o estudo permite a abordagem de um tema escasso e a possibilidade de desenvolvimento de pesquisas futuras.

\section{Referências}

1. Parada-Sanchez MT, Chu EY, Cox LL, Undurty SS, Standley JM, Murray JC, et al.
Disrupted IRF6-NME1/2 complexes as a cause ofcleftlip/Palate. J Dent Res. 2017;96:1330-8.

2. Burg ML, Chai Y, Yao CA, Magee W, 3rd, Figueiredo JC. Epidemiology, etiology, andtreatmentofisolatedcleftpalate. Front Physiol. 2016;7:67.

3. Kummet CM, Moreno LM, Wilcox AJ, Romitti PA, DeRoo LA, Munger RG, et al. Passive smoke expo2017sure as a riskfactor for oral clefts-Alargeinternationalpopulation-

basedstudy. Am J Epidemiol. 2016;183:834-41.

4. Angulo-Castro E, Acosta-Alfaro LF, Guadron-Llanos AM, Canizalez-Román A, Gonzalez-Ibarra F, Osuna-Ramírez I, et al. Maternal

riskfactorsassociatedwiththedevelopmentofcleftli pandcleftpalate in Mexico: A casecontrolstudy. Iran J Otorhinolaryngol. 2017;29:189-95.

5. Rodrigues $\mathrm{R}$, Fernandes $\mathrm{MH}$, Monteiro $A B$, Furfuro R, Sequeira T, Silva CC., \& Manso M C. SPINA classificationofcleftlipandpalate: A suggestion for a complement. Archives de Pédiatrie.2018;5(7);439-441.

6. Mossey PA, Little J, Munger RG, Dixon MJ, Shaw WC. Cleftlipandpalate. The Lancet. 2009; 374(9703):1773-1785.

7. Dixon MJ, Marazita ML, Beaty TH and Murray JC . Cleftlipandpalate: understandinggeneticandenvironmentalinfluence s. Nat RevGenet. 2011; 12(3); 167-178.

8. Freitas JA de S, Neves LT, Almeida ALPF, Garib DG, Trindade-Suedam IK, Yaedú RYF, et al. Rehabilitativetreatmentofcleftlipandpalate:

experienceofthe Hospital for Rehabilitationof Craniofacial Anomalies/USP (HRAC/USP) - Part 1: overall aspects. JournalofApplied Oral Science. 2012; 20(1), 9-15.

9. Zeraatkar M, Ajami S, Nadjmi N, Faghihi SA., \&Golkari $\quad$ A. $\quad A$ qualitativestudyofchildren'squalityoflife in thecontextof living withcleftlipandpalatePediatric Health, Medicine andTherapeutics. 2019; 10: 1320.

10. Silva FC, Oliveira TM, Almeida ALPF, Bastos RS, Neppelenbroek KH, \& Soares S. ImpactofTemporomandibularDisordersandSlee pBruxismon Oral Health-RelatedQualityof Life ofIndividualsWith Complete CleftLipandPalate. Journalof Craniofacial Surgery. 2018; 29(6):15051508.

11. Nunes LM. Prevalence of clef lip and 
palate and the notification in the information system [dissertação]. Campinas: UniversidadeEstadual de Campinas; 2005.

12. Grosen D, Chevrier C, Skytthe A, Bille C, Molsted K, Sivertsen A, et al. A cohort study of recurrence patterns among more than 54,000 relatives of oral cleft cases in Denmark: support for the multifactorial threshold model of inheritance. J Med Genet. 2010; 47:162-8.

13. Becker $M$, Svensson $H$, Kallen B. Birth weight, body length, and cranial circumference in newborns with cleft lip or palate. Cleft Palate Craniofac J. 1998; 35:255-61.

14. Wyszynski DF, Sarkozi A, Vargha P, Czeizel AE. Birth weight and gestational age of newborns with cleft lip with or without cleft palate and with isolated cleft palate. J ClinPediatrDent. 2003; 27:185-90.

15. Blanco $\mathrm{R}$, Chakraborty $\mathrm{R}$, Barton SA, Carreño $H$, Paredes $M$, Jara $L$, et al. Evidence of a sex-dependent association between the MSX1 locus and nonsyndromic cleft lip with or without cleft palate in the Chilean population. Hum Biol. 2001; 73:81-9.

16. Josiane S, Raskin S. Estudo clínico e epidemiológico de fissuras orofaciais. J. Pediatr. (Rio J.) 2013; 89(2): 137-144.

17. Nardi, Camila Guedes de Azevedo, and Maria de Lourdes MerighiTabaquim. " Avaliação neuropsicológica em crianças com fissura de lábio e palato". Anais do VIII Congresso da Sociedade Brasileira de Psicologia Hospitalar. 2011.

18. McGrew, K. S. CHC. TheoryandtheHumanCognitiveAbilities Project: Standingontheshouldersofthegiantsofpsychomet ricintelligenceresearch. Intelligence. 2009; 37(1), 1-10.

19. Cavalini, SFS, et al. "Inteligência: Efeito do tipo de escola e implicações na normatização das escalas Wechsler para crianças." Temas em Psicologia 23.2 (2015): 493-505.

20. Flores-Mendoza, C. E., \& Nascimento, E. Condição cognitiva de crianças de zona rural. Estudos de Psicologia (Campinas).2007; 24(1), 1322.

21. Gomes, CMA. Avaliando a avaliação escolar: Notas escolares e inteligência fluida. Psicologia em Estudo. 2010; 15(4), 841-849.

22. ACOBSEN, Geise $M$. et al. Qual é a participação de fatores socioeconômicos na inteligência crianças? NeuropsicologiaLatinoamericana, Calle, v. 5 , n. 4, p. 32-38, 2013.

23. Raposo-do-Amaral, C. E., Kuczynski, E., \& Alonso, N. Qualidade de vida de crianças com fissura labiopalatina: análise crítica dos instrumentos de mensuração. Revista Brasileira de Cirurgia Plástica.2011; 26(4), 639-644.

24. de Lima, EVC, Salles, AG, Marinho, BBA, Borba, RCC, Chaves, DS.,\& Marques, R. V. D. de A. Análise cognitiva e qualidade de vida em crianças portadoras de fissura orofacial não sindrômica: estudo caso controle. Revista Eletrônica Acervo Saúde.2020; 12(3), e2712.

25. BARBOSA-RESENDE $W$, et al. PsychometricpropertiesoftheAutoquestionnaireQ ualite de Vie Enfant Image (AUQEI) appliedtochildrenwith cerebral palsy. Plosone, 2015;10(2): e011564.

26. TANNURE PN, et al. Measuringtheimpactofqualityoflifeofchildrentrea ted for orofacial clefts: a case-controlstudy. JournalofClinicalPediatricDentistry, 2013; 37(4):381-4.

27. BELUCI ML, GENARO KF. Qualidade de vida de indivíduos com fissura labiopalatinapré e pós-correção cirúrgica da deformidade dentofacial. Revista da Escola de Enfermagem da USP, 2016; 50(2); 217-223.

\section{Endereço para Correspondência}

Centro Universitário - UNIFTC

Praça José Bastos, 55 - Osvaldo Cruz, Itabuna - BA

CEP.: 45600-080

E-mail: enfermagemleandro@yahoo.com.br

Recebido em 27/03/2020

Aprovado em 12/05/2020

Publicado em 18/01/2021 